\section{Regionalanästhesie als Alternative zur Allgemein- und zur Tumeszenz- lokalanästhesie bei der operativen Deckung von ausgedehnten Ulcera cruris}

T. Waldmann ${ }^{1}$, Susanne Suckow ${ }^{1}$, D. Mischke ${ }^{1}$, J. M. Pönnighaus ${ }^{1}$, F. Hendrich², L. Kowalzick ${ }^{1}$

${ }^{1}$ Klinik für Hautkrankheiten und Allergologie der Vogtlandklinikum Plauen GmbH (Leitender Arzt: Priv.-Doz. Dr. med. habil. L. Kowalzick)

${ }^{2}$ Klinik für Anaesthesie und Intensivtherapie der Vogtlandklinikum Plauen GmbH (Leitender Arzt: Dr. med. F. Hendrich)
Zusammenfassung. Bei einem großen Teil der Patienten mit venös bedingten Ulzera können aufgrund hohen Alters, Übergewichts und Begleiterkrankungen Allgemeinanästhesien nur unter einem erhöhten Risiko durchgeführt werden. Andererseits ist es schwierig, bei ausgedehnten Befunden und ausgeprägter Sklerose des Unterhautgewebes (Dermatoliposklerose) mit der Tumeszenzlokalanästhesie eine befriedigende Anästhesie zu erreichen. Als Alternative bieten sich Regionalanästhesieverfahren an. Am Beispiel einer 82-jährigen Patientin stellen wir mit dem kombinierten Femoralis-Ischiadicus-Block eine zumindest in dermatologischen Kreisen weitgehend unbekannte, schonende Regionalanästhesiemöglichkeit dar.

Regional Anaesthesia as an Alternative to General Anaesthesia or Subcutaneous Infusion Anaesthesia for the Operative Therapy of Large Venous Stasis Ulcers. A large proportion of patients with venous stasis ulcers are elderly and/or have significant co-morbidity. In such patients general anaesthesia can only be performed accepting an increased risk. On the other hand, subcutaneous infusion anaesthesia may not be satisfactory if ulcers are large and if there is marked dermatoliposclerosis. We consider regional anaesthesia an alternative. Suitable for the operative therapy of venous ulcers is in particular a combination of a block of the N. femoralis and the N. ischiadicus. Using this technique a large nearly circumferential venous stasis ulcer could be completely healed with meshed skin grafts in a 82 year old woman.

\section{Einleitung}

Seit einigen Jahren hat sich die operative Deckung bei gegenüber konservativen Therapieversuchen resistenten Ulzera immer mehr durchgesetzt [1]. Ein häufiges Problem, das vor allem bei älteren und multimorbiden Patienten auftritt, ist eine suffiziente Anästhesie zu erzielen. Während für Allgemeinanästhesien aufgrund von Begleiterkrankungen ein erhöhtes Risiko besteht, lassen sich mit der Lokalanästhesie oder der Tumeszenzlokalanästhesie besonders bei ausgedehnten Ulzera

Akt Dermatol 2001; 27: 262-264

(c) Georg Thieme Verlag Stuttgart · New York ISSN 0340-2541 und bei ausgeprägter Dermatoliposklerose häufig keine zufriedenstellenden Betäubungen des OP-Gebietes erreichen.

\section{Methode}

1. 3-in-1-Block (Blockade des N. femoralis, des N. obturatorius und des N. cutaneus femoris lateralis): Markierung des Einstichpunktes lateral der A. femoralis ungefähr $1,5 \mathrm{~cm}$ unterhalb des Leistenbandes (Abb. 1). Nach sorgfältiger Desinfektion Einstich der mit einem Stimulator verbundenen Nadel (Abb. 2). Stimulation mit dem Nervenstimulationsgerät (Stimuplex S) mit 1,0 mA bis zum Auslösen von Kontraktionen im $M$. rectus femoris. Die Stimulationsintensität sollte schrittweise bis zu einer Stärke von $0,4 \mathrm{~mA}$ reduziert werden. Wenn sich damit noch Kontraktionen auslösen lassen, kann man von einer optimalen Platzierung der Nadelspitze ausgehen. Anschließend erfolgt langsam die Injektion von ca. $30 \mathrm{ml}$ Xylonest ${ }^{\circledR} 1 \%$. Dabei sollte distal des Injektionspunktes komprimiert werden, um so ein Aufsteigen des Lokalanästhetikums zu erreichen und auch den N. obturatorius für die medialen Anteile der Haut des Oberschenkels sowie den N. cutaneus femoris lateralis für den lateralen Anteil der Oberschenkelhaut mit zu betäuben [2].

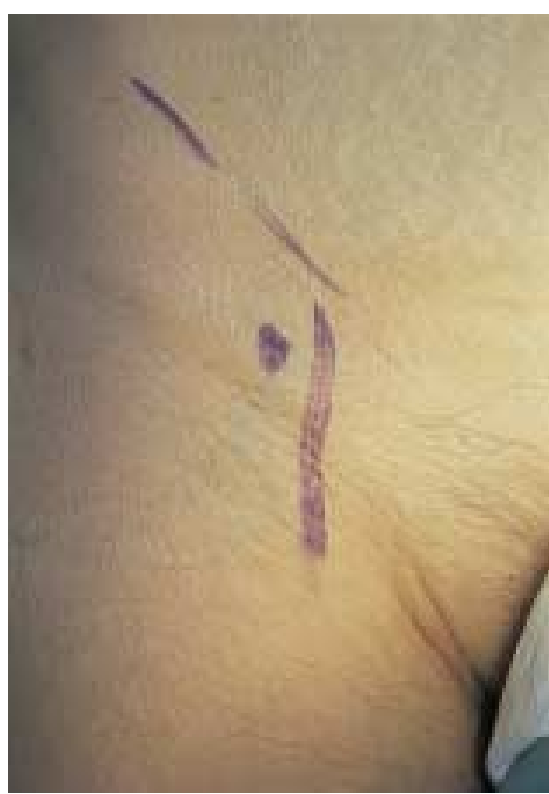

Abb. 1 3-in-1Block: Markierung des Einstichpunktes. 


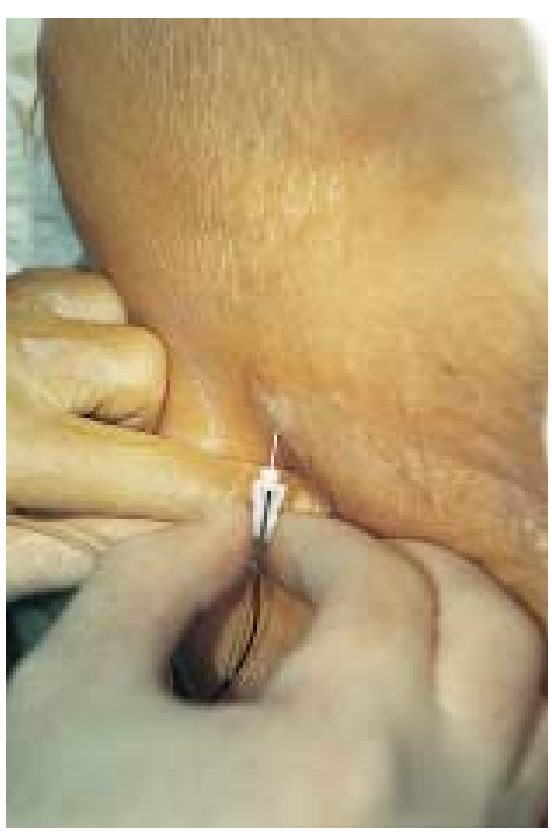

Abb. 2 3-in-1Block: Einstich der mit einem Nervenstimulator verbundenen Nadel.

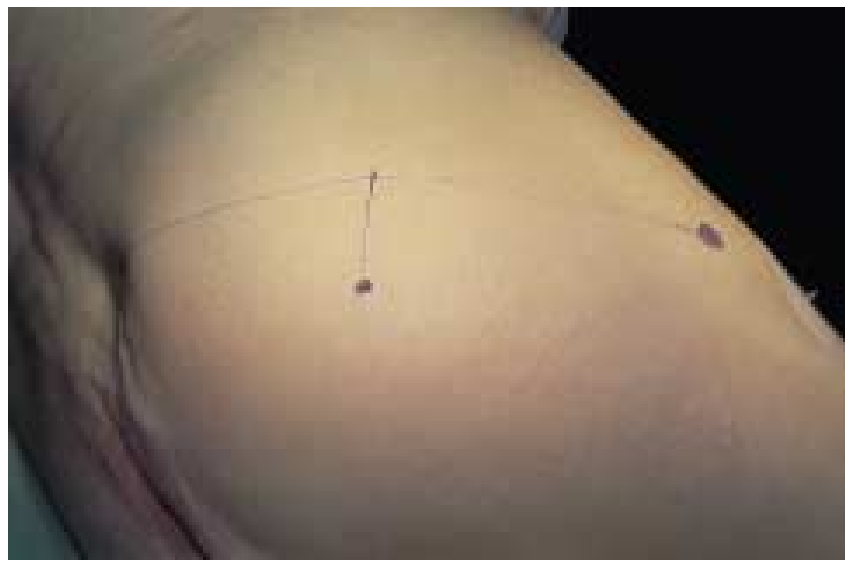

Abb. 3 Markierung des Einstichpunktes für die Blockade des N. ischiadicus.

2. Dorsale transgluteale Ischiadicusblockade nach Labat: Für die Blockade des N. ischiadicus muss der Patient in Seitenlage gebracht werden, wobei das zu betäubende Bein oben liegen und im Hüftgelenk in einem Winkel von ca $135^{\circ}$ zur Körperachse, im Kniegelenk ungefähr $90^{\circ}$ gebeugt sein sollte. Als Leitstrukturen dienen die Spina iliaca posterior superior und der Trochanter major. In der Mitte einer Verbindungslinie zwischen beiden Punkten fällt man das Lot und markiert einen Punkt ca. 4-5 cm weiter dorsal (Abb.3). Nach gründlicher Desinfektion erfolgt der Einstich der Nadel (ca. $10-15 \mathrm{~cm}$ tief). Die Stimulation erfolgt analog wie für den $\mathrm{N}$. femoralis beschrieben, jedoch sollen Kontraktionen am Unterschenkel ausgelöst werden. Für die Blockade des N. ischiadicus werden ungefähr $20 \mathrm{ml}$ Xylonest ${ }^{\circledR} 1 \%$ benötigt [2,3].

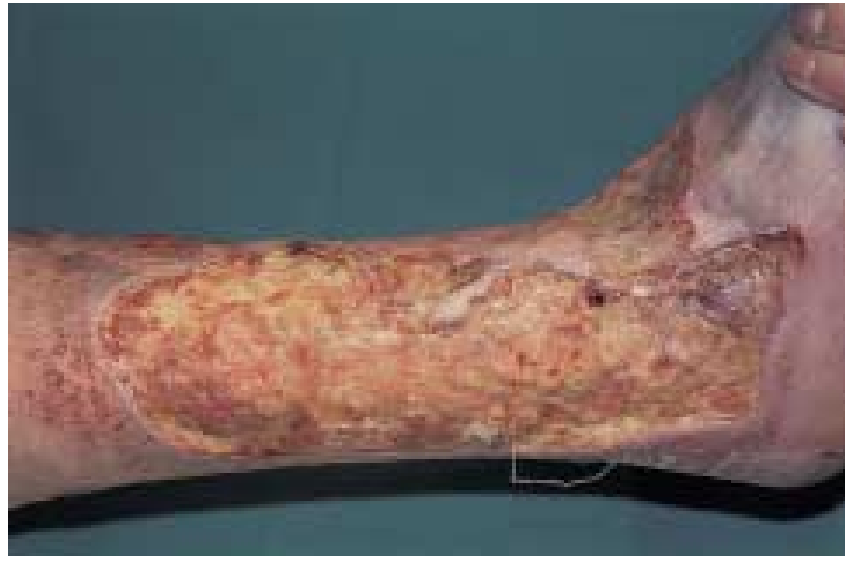

Abb. 4 Präoperativer Befund: Ausgedehntes, fast die gesamte Zirkumferenz einnehmendes, schmierig belegtes Ulkus am rechten Unterschenkel.

\section{Kasuistik}

\section{Anamnese}

Die 82-jährige Patientin berichtete, dass sie seit 2 Jahren „offene Beine“ habe. Die Ulzera seien sehr schmerzhaft. Verschiedene konservative Therapieversuche seien ohne Effekt geblieben. Weiterhin gab die Patientin an, dass bei ihr viele Allergien bekannt seien. Ungefähr einen Monat vor der stationären Aufnahme musste die Patientin wegen einer Sepsis und einem prärenalen Nierenversagen in der Medizinischen Klinik unseres Hauses behandelt werden.

\section{Hautbefund}

Bei Aufnahme sahen wir am rechten Unterschenkel mehrere ineinander übergehende, fast den gesamten Umfang des Unterschenkels einnehmende, schmierig gelblich belegte Ulzera (Abb. 4). Lediglich an der Tibiavorderkante bestand ein schmaler Streifen mit erhaltener Epidermis. Der gesamte Unterschenkel war sklerotisch im Sinne einer Dermatosklerose verändert. Weiterhin bestand am linken Unterschenkel ein $2,5 \times 3,5 \mathrm{~cm}$ großes, relativ sauberes Ulkus. Die Venen an beiden Beinen waren varikös verändert.

\section{Diagnosen}

Ulcera crurum bei chronisch venöser Insuffizienz, multiple Typ-IV-Sensibilisierungen,

Herzinsuffizienz,

Z. n. Sepsis und prärenalem Nierenversagen.

\section{Befunde}

Labor: (SI)

Leukozyten 19,0; Hämoglobin 6,1; Hämatokrit 0,30; Kalium 2,71; Kreatinin 128, Harnstoff 13,6; Harnsäure 614; CrP 79,0

Die sonstigen Routinelaborparameter waren ohne wesentlichen pathologischen Befund.

Wundabstrich (Ulkus):

Pseudomonas aeruginosa mit multiplen Resistenzen, sensibel gegenüber Imipenem und Meropenem. 


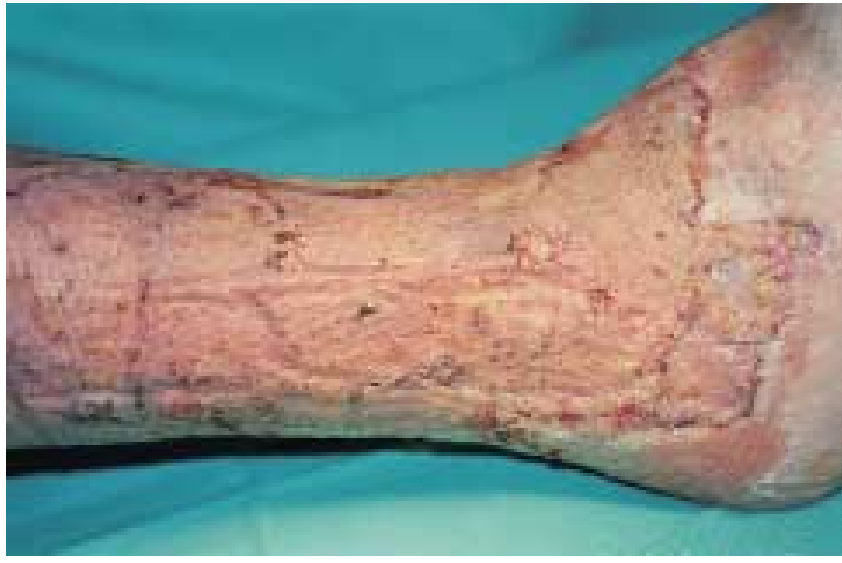

Abb. 5 Abgeheiltes Ulkus 5 Wochen postoperativ.

Dopplersonographie:

Arteriell: Unauffällige arterielle Durchblutungsverhältnisse. Venös: Komplette Insuffizienz der Vena saphena magna Grad IV rechts.

Isolierte Insuffizienz der Cockettschen Perforanten I und II bei Z.n. Vena saphena magna Stripping links.

Epikutantestung (Standard, Arzneistoffe, Desinfektions- und Konservierungsstoffe, Salbengrundlagen und Emulgatoren): positiv waren: Wollwachsalkohole, Thiuram-Mix, DuftstoffMix, Benzoylperoxid, Chlorcresol, Chlorxylenol, Butylhydroxyanisol (BHA), tert.-Butylhydrochinon, Amerchol L101.

\section{Therapie und Verlauf}

Nach Anästhesie des rechten Beines durch eine Kombination eines 3-in-1-Blockes mit einer Blockade des N. ischiadicus führten wir die Deckung der Ulzera mittels vom rechten Oberschenkel entnommener, gemeshter Spalthaut durch. Weder bei der Reinigung der Ulzera mittels Handdermatom noch bei der Spalthautentnahme klagte die Patientin über Schmerzen. Die Operation verlief komplikationslos. Die postoperative Antibiose erfolgte aufgrund der multiplen Resistenzen des Keimes mit Imipenem. Das Transplantat heilte nahezu vollständig ein, kleine Restdefekte wurden in einer zweiten Sitzung in örtlicher Betäubung mit Reverdinläppchen verschlossen. Bei Entlassung waren die Ulzera komplett abgeheilt (Abb.5). Die Patientin wurde mit Kompressionsstrümpfen Klasse II versorgt und ein Pflegedienst wurde organisiert.

\section{Diskussion}

Ulcera crurum als Folge einer chronisch venösen Insuffizienz oder eines postthrombotischen Syndroms stellen eine der häufigsten Erkrankungen unserer Gesellschaft dar. So erleiden ungefähr $1 \%$ aller Männer und 2\% aller Frauen zumindest einmal im Leben ein venöses Ulkus [3]. Mit konservativen Therapieansätzen wie z.B. Hydrokolloidverbänden und Alginaten und gleichzeitiger Kompressionstherapie kann bei einem Teil der Patienten eine Abheilung in relativ kurzer Zeit erreicht werden. Bei therapieresistenten Verläufen hat sich in den letzten Jahren immer mehr die operative Deckung der Ulzera durchgesetzt $[1,4,5]$. In unserer Klinik hat sich die Shave-Therapie mit anschließenden Spalthauttransplantationen bewährt [6], wobei die gemeshten Transplantate lediglich mit
Pflasterspray am Ulkusgrund fixiert werden [7]. Damit können neben hohen Abheilungsquoten auch gute Langzeitergebnisse erreicht werden [8] vorausgesetzt, dass nach Abheilung eine konsequente Kompressionsbehandlung oder eine operative Sanierung der insuffizienten Venen durchgeführt wird.

Bei den häufig älteren und multimorbiden Patienten bestehen oft erhebliche Einschränkungen bezüglich der Narkosefähigkeit. Mit der seit einigen Jahren vermehrt durchgeführten Tumeszenzlokalanästhesie (TLA) [9] steht eine relativ schonende Betäubungsmöglichkeit zur Verfügung, die sich auch bei der Ulkusdeckung bewährt hat. Probleme zeigten sich jedoch bei sehr ausgedehnten Ulzera, da man schnell die vom Hersteller angegebene Höchstdosis von $7 \mathrm{mg} / \mathrm{kg}$ KG Prilocain (Xylonest ${ }^{\circledR}$ ) [10] erreicht. Weiterhin stellte es sich bei uns als problematisch heraus, bei Ulzera mit einer ausgeprägten Dermatosklerose z.B. im Rahmen eines postthrombotischen Syndroms eine zufriedenstellende Schmerzfreiheit zu erzielen.

Mit der Kombination von 3-in-1-Block und N. ischiadicusBlockade steht eine weitere, zumindest in der dermatologischen Literatur weitgehend unbekannte, Anästhesiemöglichkeit zur Verfügung. Bei korrekter Durchführung kann mit dieser Methode eine sehr gute Schmerzfreiheit bei im Vergleich zu anderen Verfahren geringem Aufwand und geringen Belastungen für den Patienten erzielt werden. So konnten die ausgedehnten Ulzera bei unserer Patientin problemlos operativ saniert werden, ohne dass ein erhöhtes Risiko einer Allgemeinanästhesie für diesen Elektiveingriff eingegangen werden musste.

\section{Literatur}

${ }^{1}$ Korstanje MD. Venous stasis ulcers - diagnostic and surgical considerations. Dermatol Surg 1995; 21: 635-640

${ }^{2}$ Niesel HC. Regionalanästhesie - Lokalanästhesie, regionale Schmerztherapie. Stuttgart: Thieme, 1994

${ }^{3}$ Labat G. Regional anesthesia: Its technic an clinical application. Philadelphia: W. B. Saunders, 1994

${ }^{4}$ Orfanos CE, Garbe C. Therapie der Hautkrankheiten. Berlin, Heidelberg: Springer, 1995

${ }^{5}$ Christiansen J, Lorens EK, Tegner E. Pinch grafting of leg ulcers. Acta dermato-venerologica 1997; 77: 471 - 473

${ }^{6}$ Thiele H, Kohler U. Das Ulcus cruris venosum. Langenbecks Arch Chir 1997; 2: $513-516$

${ }^{7}$ Schmeller W, Gaber Y. Behandlung therapieresistenter venöser Ulzera mittels Shave-Therapie. Dt Ärzteblatt 2000; 97: 2464-2467

${ }^{8}$ Ghosh JS, Kumar K, Gilbert PM. Opsite spray: its use for fixation of meshed skin grafts. Simple and low tech. Burns 1997; 23: 601 -603

${ }^{9}$ Dunn JM, Cosford EJ, Kernick VFM, Campbell WB. Surgical treatment for venous ulcers: is it worthwhile. Ann R Coll Surg Engl 1995; 77: $421-424$

${ }^{10}$ Breuninger H, Caroli JW. Subkutane Infusionsanästhesie (SIA) mit durch Ringer-Lösung verdünntem Prilocain. Hautarzt 1998; 49: $709-713$

${ }^{11}$ Sommer B, Augustin M, Schöpf E, Sattler G. Tumeszenz-Lokalanästhesie. Dt Ärzteblatt 2001; 98: 545 - 548

\section{Dr. med. Thomas Waldmann}

Vogtlandklinikum Plauen GmbH

Klinik für Hautkrankheiten und Allergologie

Maximilian-Kolbe-Weg 1a

08529 Plauen 